\section{$\S 14$. Asymmetrical Bifurcation of the Radial Electric Field Structure Induced by Electrode Biasing in Tokamaks}

Kasuya, N. (Sch. Science, Univ. Tokyo)

Itoh, K.

Takase, Y. (Sch. Frontier Sciences, Univ. Tokyo)

The transition from the L-mode to the $\mathrm{H}$-mode is a bifurcation phenomenon. The radial electric field structure distinguishes the $\mathrm{L} / \mathrm{H}$ mode, and spatially non-constant structures are taken in the $\mathrm{H}$-mode. We extend the previous $\mathrm{H}$-mode bifurcation model in tokamaks to include the effect of electrode biasing. The plasma nonlinear response is the origin of structural bifurcation, and electrode biasing acts as an additional driving term for structure formation. It shows a unique asymmetric feature between positive and negative biasing.

A model equation for the radial electric field structure is a combination of Poisson's equation and the charge conservation law,

$$
\frac{\partial}{\partial t} E_{\mathrm{r}}=-\frac{1}{\varepsilon_{0} \varepsilon_{\perp}}\left(J_{\text {visc }}+J_{\mathrm{r}}+J_{\text {erbit }}-J_{\text {ext }}\right),
$$

where $J_{\text {visc }}$ is the current driven by shear viscosity, $J_{\mathrm{r}}$ is the local current, $J_{\text {orbit }}$ is the current driven by ion orbit losses, $J_{\text {ext }}$ is the current driven into the electrode by the external circuit, $\varepsilon_{0}$ is the vacuum susceptibility, and $\varepsilon_{\perp}$ is the dielectric constant of a magnetized plasma. $J_{\text {visc }}$ term acts as a diffusion term due to anomalous shear viscosity.

Various structures with multiple peaks are obtained for the same boundary condition.1) The model equation has two nonlinear terms, $J_{\mathrm{r}}$ and $J_{\text {orbit. }}$. Their balancing explains the $\mathrm{H}$-mode transition mechanism and an asymmetrical bifurcation feature with respect to the electrode current term.2) The ambipolar radial electric field $\left(X_{\mathrm{a}}\right)$ varies in accordance with the pressure gradient, and it affects the dependency of the radial current and can give an asymmetric property as well. For the positive biasing case, various solitary solutions can exist, and a radial electric field transition can take place even though the profiles of plasma parameters become steeper (when $\left|X_{\mathrm{a}}\right|$ becomes larger). On the contrary for the negative biasing case, the two nonlinear terms have opposite signs and increase the number of spatially constant solutions for adequate conditions depending on the ambipolar radial electric field, the distance between the electrode and the limiter, and so on. Figure 1 shows a phase diagram in the $X_{\mathrm{a}}-V_{\text {ext }}$ space, where $V_{\text {ext }}$ is the applied biasing voltage. The threshold voltage for hard transition is given by the boundaries of the shaded regions. The boundaries neighboring $\mathrm{S}$ and $\mathrm{T}$ give the forward transition threshold and the backward transition threshold, respectively. As the ambipolar radial electric field increases, transitions still take place for positive biasing, but do not take place for negative biasing. When the distance between the electrode and the limiter is large, the competition between the two nonlinear terms gives a larger number of solutions for adequate conditions.

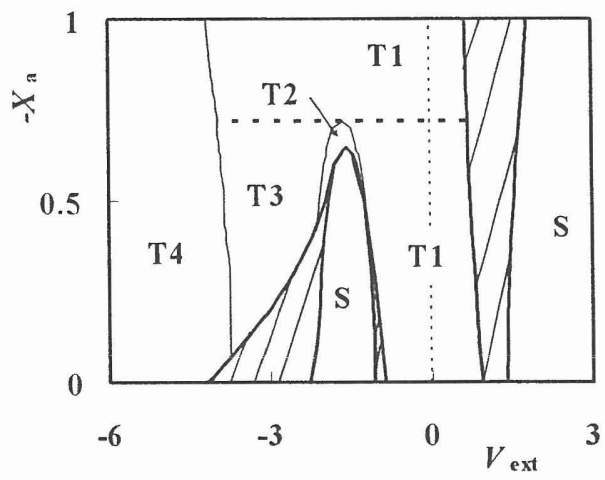

Fig.1 Phase diagram of the radial electric field structure. $\mathrm{S}$ and $\mathrm{T}(\mathrm{T} 1 \sim \mathrm{T} 4)$ represent regions where a solitary solution and a spatially constant solution exist, respectively. The shaded regions indicate where both solutions can exist and give hysteresis. In this figure only solitary solutions that have one peak are considered.

\section{Reference}

1) Kasuya, N., et al., Plasma Phys. Control. Fusion. 44 (2002) A287

2) Kasuya, N., et al., Plasma Phys. Control. Fusion 45 (2003) 183 